\title{
THERMO-MECHANICAL SCREENING TESTS TO QUALIFY BERYLLIUM PEBBLE BEDS WITH NON-SPHERICAL PEBBLES
}

\author{
Joerg Reimanna ${ }^{a}$ Benjamin Fretz ${ }^{b}$, Simone Pupeschic \\ aIKET, Karlsruhe Institute of Technology, Karlsruhe, Germany \\ ${ }^{b} \mathrm{KBHF} \mathrm{GmbH}$, Eggenstein-Leopoldshafen, Germany \\ CIAM, Karlsruhe Institute of Technology, Karlsruhe, Germany
}

\section{- Objectives}

- In present ceramic breeder blankets, pebble-shaped beryllium is used as a multiplier. As candidate material, spherical pebbles with diameters of $\mathrm{d} \approx$ $1 \mathrm{~mm}$ are considered.

- Non-spherical particles are of significant economical interest. Except of packing factors ${ }^{1}$, no thermo-mechanical pebble bed data exist for nonspherical beryllium grades.

- Qualification tests were performed in helium atmosphere at ambient temperature: Uniaxial Compression Tests (UCTs) combined with the Hot Wire

\section{- Experimental}

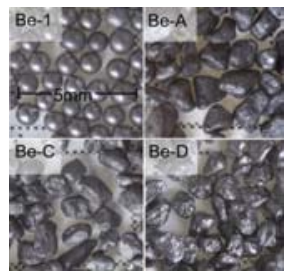

\section{Investigated beryllium grades:}

Be-1: spherical $1 \mathrm{~mm}$ pebbles, NGK, Japan

Be-A, Be-C: $2.5 \mathrm{~mm}$ pebbles, different grain sizes, Bochvar, Russia, Be-D: $2 \mathrm{~mm}$ pebbles, Materion, USA

UCT and HWT experimental set-up: Only $\approx 120 \mathrm{~cm}^{3}$ of nonspherical beryllium grades were available. This resulted in a small set-up with a somewhat reduced measurement accuracy, "screening tests". Therefore, the comparison with the spherical beryllium pebbles was important. Technique (HWT) to measure the thermal conductivity $k$

\section{Hot Wire Modelling}

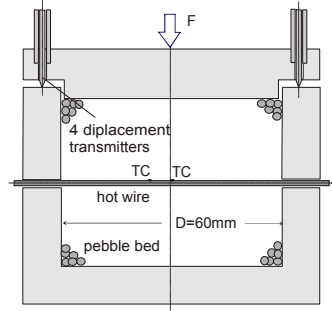

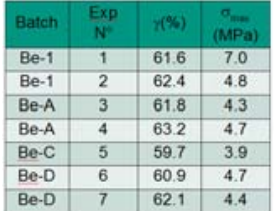

Experimental parameters: packing factors $\gamma$ and maximum uniaxial stresses $\sigma$

The HW Technique is a standard technique for thermal conductivity $\mathrm{k}$ measurements of materials with low $\mathrm{k}$ values in large containers. Both requirements are not fulfilled in the present case. Therefore, a detailed modelling of the HWT is required for the interpretation of the HW signal.

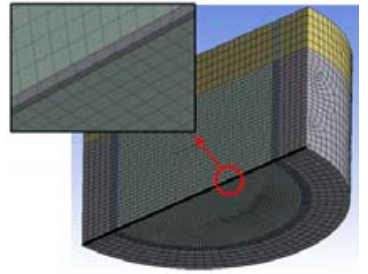

a)
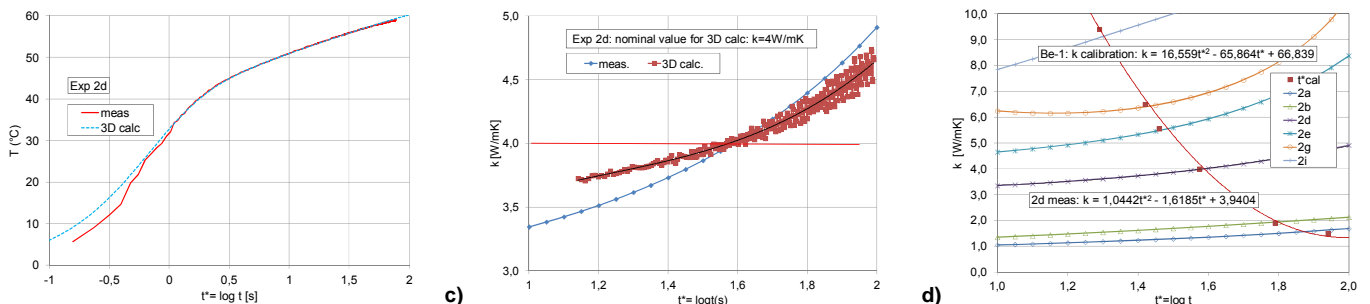

a) 3-D transient analyses with the FE ANSYS code were performed modelling in detail the HW (with inner structure) and the container.

b) A nominal value for the pebble bed thermal conductivity has been assumed, and then, the measured curve is approached by varying the HTCs at the HW and the container walls. After a first period of time, the slope of an ideal HW temperature curve becomes constant (half-log plot). This is not the case for both the measured and calculated signal.

c) Because of the varying slope, the measured and calculated values of $k$ are not constant. As correct value $t^{*}$ that value is taken where measured and calculated values agree (iteration process) d) This procedure is carried out for different values of $\mathrm{k}$ and a calibration curve is obtained. Different curves are determined for spherical and non-spherical pebble beds.

\section{- Experimental Results}

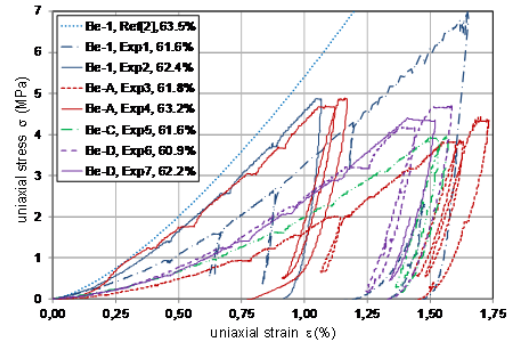

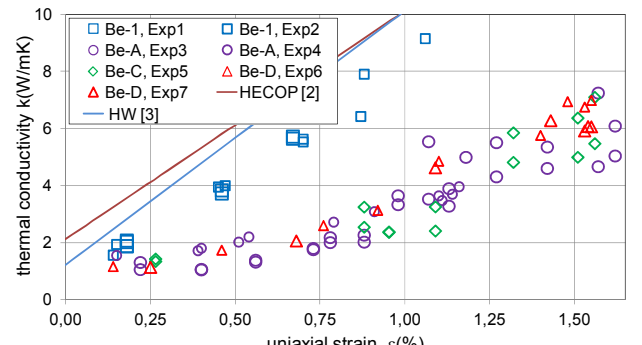

$\uparrow$ HWT results: $k=f(\varepsilon)$. For non-spherical pebbles, $k$ is distinctively smaller than for spherical ones, mainly caused by the softer $\sigma-\varepsilon$ relation. No differences exist between the different non-spherical grades. Again, $\mathrm{k}$ is fairly linear dependent on $\varepsilon$ as found previously $y^{2,3}$.

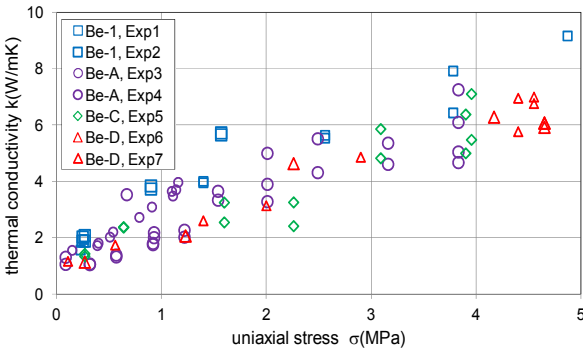

HWT results: $k=f(\sigma) . k$ for spherical pebbles is at the upper bound of the data which might be caused by different sizes of generated contact surfaces during compression. decreasing packing factor $\gamma$, the pebble beds become (larger strain $\varepsilon$ for a given stress $\sigma$ ). Be-1 and Exp 4 with Be-A show the stiffest behaviour, the values are, however, below the correlation obtained with a larger experimental set-up².

\section{- Conclusions}

Compared to spherical pebble beds, the thermal conductivity for non-spherical pebble beds is lower caused by i) the softer bed behaviour (smaller stress $\mathrm{s}$ for a given strain e value), and, ii) the generation of smaller contact surfaces because of the non-regular shape.

- For blanket operation, the pebble bed strain is the primary parameter; for softer pebble beds the anticipated increase of the thermal conductivity during heating-up is smaller because of the reduced build-up of thermal stresses. 